\title{
EEG Characteristics of Adolescents Raised in Institutional Environments and Their Relation to Psychopathological Symptoms
}

\author{
Jorge Carlos Hevia-Orozco' ${ }^{1}$, Araceli Sanz-Martin ${ }^{2 *}$ \\ ${ }^{1}$ Instituto de Neurobiología, Universidad Nacional Autónoma de México, Querétaro, México \\ ${ }^{2}$ Instituto de Neurociencias, Universidad de Guadalajara, Guadalajara, México \\ Email: *araceli.sanz.martin@gmail.com
}

How to cite this paper: Hevia-Orozco, J.C. and Sanz-Martin, A. (2018) EEG Characteristics of Adolescents Raised in Institutional Environments and Their Relation to Psychopathological Symptoms. Journal of Behavioral and Brain Science, 8, 519-537. https://doi.org/10.4236/jbbs.2018.810032

Received: August 27, 2018

Accepted: September 27, 2018

Published: September 30, 2018

Copyright (c) 2018 by authors and Scientific Research Publishing Inc. This work is licensed under the Creative Commons Attribution International License (CC BY 4.0).

http://creativecommons.org/licenses/by/4.0/

\begin{abstract}
Children reared in institutional environments present emotional and cognitive affectations, as well as changes in the anatomy and functioning of certain cerebral areas, such as the prefrontal cortex. However, knowledge of the long run effect of these affectations remains scarce. Therefore, this study was designed to correlate the cortical electroencephalographic (EEG) and psychopathological parameters. Resting EEG from 10 institutionalized (IA) and 10 never-institutionalized adolescents (NIA) was obtained and this was parametrically correlated with the intensity of symptoms of depression, post-traumatic stress disorder (PTSD) and anxiety. Results showed that the IA group showed higher indices of depression, anxiety and PTSD, accompanied by low relative power (RP) in the fast bands, high activity in the slow bands in frontal areas, and higher alpha2 RP in temporal areas. In addition, IA showed higher EEG-correlations among frontal areas but lower EEG-correlations among prefrontal, parietal and temporal areas in the gamma band. We also found correlations between the EEG parameters and psychopathological scores. These EEG findings can contribute to improve our understanding of how stress experienced early in life may impact the development of the cortex, and how this, in turn, may predispose individuals to develop psychiatric conditions.
\end{abstract}

\section{Keywords}

Adolescents, Institutionalization, EEG Activity, Depression, Anxiety

\section{Introduction}

Institutionalization is defined as the confinement of an infant in any large con- 
gregate care facility where round-the-clock professional supervision supplants the role of family-like caregivers [1]. In this model of child-rearing, children and adolescents remain in a space of a closed or restricted character (i.e., no contact with family or community) [1]. One common characteristic of institutionalization is the general deprivation in various areas that children may suffer [2], since the quantity and quality of stimulation provided barely meet the minimum standards required to achieve basic development [3]. Indeed, some reports show that the level of stimulation is insufficient for the optimal development of various emotional and cognitive functions [4] [5]. Worse yet, this deprivation can aggravate the consequences of the stress that these children may have suffered in their birth families.

Recently, some authors have reported that institutionalized children are more prone to developing certain psychiatric conditions in the future, including symptoms of attention-deficit/hyperactivity disorder [6], depression [7], and anxiety [8]. Indeed, some earlier studies postulated the existence of a syndrome in this population that mimics diagnoses of autism [9]. This predisposition has been related to the presence of alterations in the development of certain cortical areas and their interconnectivity. The prefrontal, parietal and temporal cortices, for example, have all shown developmental alterations [6] [10] [11]. Also, the connectivity among prefrontal and posterior cortical areas was shown to be altered in post-institutionalized Rumanian children (through the uncinate fasciculus and the superior longitudinal fascicule) [12] [13].

It is well-known that adequate cerebral functioning depends on the coordinated work of several brain structures [14]; hence, it is important to examine the functional coupling or decoupling among them. One way to measure cerebral functionality is by recording electroencephalographic activity (EEG), a technique that offers researchers several advantages, since it is non-invasive and easily-applied in institutionalized environments because it does not require a laboratory setting [15]. Relative power (RP) is an index of the proportion of power in a specific frequency band at a given electrode site, while Correlation ( $r$ ) analyses of EEG signals make it possible to determine the degree of coupling-or functional synchronization-between two cerebral areas.

In relation to the effects of institutionalization on EEG, McLaughlin et al. [7] recorded the EEG activity of 6 - 30-month-old children raised in institutions and compared it to that of an age- and sex-matched control sample of community participants. They found that, compared to the participants raised with their biological families, the institutionalized children had a lower RP in the alpha band and a higher RP in the theta band in the frontal, temporal and occipital regions; results that suggest a delay in cortical maturation. Although the children raised in institutions presented more pronounced symptoms of anxiety and depression, RP was not related to those psychopathologies. Similar results from the alpha and theta bands were found previously in a study conducted with a similar sample under similar conditions, though that work also found a lower RP in the beta 
band in occipital regions of institutionalized children [11]. Using a similar protocol, Marshall et al. assessed two groups of children aged 42 months, one institutionalized, the other living in foster homes. They found that placement in foster care at an earlier age (i.e., before 24 months) was associated with increased alpha power, decreased frontal-central and frontal-temporal EEG coherences in the alpha band, and reduced frontal-central EEG coherences in beta [11]. These data suggest that foster care was associated with neurophysiological changes in the central nervous system in previously-institutionalized children, and that the extent of these changes was partly dependent on the age of placement in foster care.

These EEG findings reported for institutionalized children could be associated with psychopathological problems in later stages of life. Some psychiatric illnesses, like autism, are currently attributed, theoretically, to a lack of connectivity between the prefrontal cortex and different cortical and subcortical areas [16] [17] and with the presence of higher theta and lower alpha RP [18] [19]. However, the aforementioned studies have been conducted with institutionalized preschoolers or school-age children, so as far as we know no work has yet evaluated EEG changes in institutionalized adolescents or explored their possible association with the mood alterations that these populations often manifest.

Considering that mood modulation depends on the integrity of the prefrontal cortex and its functional interaction with other, associated cortical areas, and that institutionalization has been associated with emotional alterations in children, the aim of the present study was to correlate prefrontal, parietal and temporal EEG activity with psychopathological symptoms in institutionalized adolescents compared to community-reared adolescents.

\section{Method}

\subsection{Participants}

Participants were 10 male adolescents housed in private or government-sponsored orphanages (institutionalized adolescents, IA), who were compared to 10 never-institutionalized adolescents (NIA). All participants were right-handed, aged 13 - 16 years, with no current neurological or psychiatric treatment. They were attending middle school or high school, had IQ $\geq 70$ as evaluated by the brief form of the Wechsler Intelligent Scales [20] [21], and normal scores for attention and concentration as measured by a Spanish-language battery called NEUROPSI A-M [22]. Inclusion criteria for the study were: 1) that the IA participants had lived over $75 \%$ of their lives in the institution; and, 2) subjects in the NIA group had lived their entire lives in family with their parents. Regarding the history of the IA participants, access to each one's records in the government institutions responsible for the protection of minors was obtained. Documents in the institutions where they were living were also reviewed. This allowed us to determine that none had suffered sexual abuse, but that prior to entering the institution, $50 \%$ had been victims of physical violence, $40 \%$ had suffered physical neglect, 
and $10 \%$ had experienced psychological violence. Also, two of them were taking antidepressant medication (sertraline or fluoxetine) at the time of the evaluation.

With regard to exclusions, 4 institutionalized teenagers were not included in the final analysis due to excessive artefacts in the EEG recordings. IA participants were recruited from 4 different institutions located in 3 states in Mexico by direct invitation from their respective Principal. All institutions were registered under the Mexican Official Norms for Assistance Services for Children (Nom-167-SSA1-1997), which ensured that the adolescents' housing, clothing and food needs and health services would be fully-covered while they participated in the project. Once permission was obtained from parents or caregivers, an appointment for applying the psychometric tests was scheduled. Control group was recruited from a secondary school of one of the same states and was invited through an informational meeting with their parents. Each adolescent from control group live in a nuclear family and did not present any sign of maltreatment. Individuals of both groups were matched according to age and IQ scores. The study took along one year to be completed approximately.

The study was conducted in two sessions. During the first meeting, the attention, concentration, IQ, Depression, Anxiety and PTSD tests were applied. All EEG recording was performed in the second session at baseline with eyes open.

All procedures involved in this experiment were approved by the Ethics Committee of the Institution in accordance with the ethical standards established in the 1964 Helsinki Declaration. All participants gave their informed consent prior to inclusion. The demographic characteristics of the sample are summarized in Table 1.

\subsection{Procedure}

EEG recordings were taken at eight derivations of the International 10 - 20 System. Electrodes were placed in prefrontal, frontopolar (Fp1, Fp2) and dorsolateral (F3, F4) areas, and in temporal (T3, T4) and parietal (P3, P4) regions. EEG-recording lasted for 3 min with subjects at rest with eyes open. All derivations were referred to linked earlobes with the ground electrode placed on the forehead. Electrooculograms were recorded to detect eye-movement artefacts using a monopolar montage with electrodes placed at the outercanthi of both

Table 1. Demographic characteristics of the participants. This table represents features of the institutionalized (IA) and never-institutionalized (NIA) adolescents that were used to match both groups. IQ: Intelligent quotient (mean \pm standard error: $\mathrm{M} \pm \mathrm{SE}$ ).

\begin{tabular}{ccccccccc}
\hline \multirow{2}{*}{ Demographic data } & \multicolumn{2}{c}{ IA $(\mathrm{n}=10)$} & \multicolumn{2}{c}{ NIA $(\mathrm{n}=10)$} & \multicolumn{2}{c}{ Comparisons } & \multirow{2}{*}{ Cohen's d } \\
\cline { 2 - 7 } & $\mathrm{M}$ & S.E. & $\mathrm{M}$ & S.E. & $\mathrm{t}$ & $\mathrm{p}$ & \\
\hline Age & 14.8 & \pm 1.033 & 14.1 & \pm 0.738 & 1.74 & 0.098 & 0.379 \\
IQ & 88.9 & \pm 14.07 & 85.0 & \pm 8.62 & 0.75 & 0.46 & 0.174 \\
Months of institutionalization & 153.9 & \pm 17.09 & --- & --- & --- & -- & \\
\hline
\end{tabular}


eyes. The EEG signals were amplified digitally by a Neuroscan-Nu Amps digital amplifier (Compumedics Neuroscan), with EEG filters set at 1 and $50 \mathrm{~Hz}$. Impedance for the EEG electrodes was kept below $10 \mathrm{kV}$ using Neuroscan sintered silver chloride electrodes (Compumedics Neuroscan). EEG signals were simultaneously recorded in a computer using the Scan 4.3 Software program (Compumedics Neuroscan). The EEG epochs that were identified visually as being contaminated by noise were removed manually with the CHECASEN computer program [23]. All contaminated segments were eliminated. Later, the EEGs were analysed by the EEG Bands computer program [24], which initially calculated the Fast Fourier Transform to seven frequency bands: delta $(1.5-3.5 \mathrm{~Hz})$, theta (3.5 - 7.5 Hz), alpha1 (7.5 - 10.5 Hz), alpha2 $(10.5-13.5 \mathrm{~Hz})$, beta1 $(13.5-19.5$ $\mathrm{Hz})$, beta2 $(19.5-30 \mathrm{~Hz})$, and gamma $(31-50 \mathrm{~Hz})$. Fast Fourier transformed and EEG relative power (RP) was obtained with $0.5 \mathrm{~Hz}$ resolution from $1-50 \mathrm{~Hz}$ for each participant. The correlation spectrum ( $r$ ) was then calculated based on the autospectra and cross-spectrum of the signals to produce values between -1 and +1 . Correlation spectra at 0 delay were obtained for each frequency band using amplitude values by means of Pearson product-moment coefficients to obtain intrahemispheric and interhemispheric correlations between derivations in the right (Fp2, F4, T4, P4) and left (Fp1, F3, T3, P3) hemispheres. RP values were transformed to logarithms, while correlation values were transformed to Fisher's z-scores to approximate them to a normal distribution before conducting the statistical procedure. Z-score calculations were based on individual scores.

\subsection{Measures}

The Children's Depression Inventory (CDI) Spanish version was applied [25]. This is a 27-item scale that describes feelings or thoughts in adolescents during the previous two weeks related to depression or dysthymic disorder. The format for responses is based on 1,2,3-scales according to the extent of depression reflected in their choice of one of the 3 response options offered for each item; e.g., one sample item presents these three options: "I'm seldom sad" 1), "I'm frequently sad" 2), and "I'm always sad" 3). This scale assesses a total score based on two sub-domains: dysphoria and low self-esteem. Significant clinical symptomatology based on the total scale and the two subscales is considered when scores are 65 or higher.

The Children's Post-Traumatic Stress Scale-Spanish version (CPSS) [26] was used to determine the presence of Post-Traumatic Stress Disorder (PTSD). This scale is based on the diagnostic criteria of the DSM-IV-TR and consists of 17 questions that are answered on a 1 - 4 Likert scale, depending on the frequency of symptoms. According to the DSM-IV-TR, the presence of PTSD is considered when at least 1 symptom of re-experimentation, 2 symptoms of arousal, and 3 symptoms of avoidance occurred during the previous month. PTSD is judged to be present with the appearance of 3 symptoms on the Likert scale. Subjects are 
instructed to indicate if they have had any thoughts in the previous two weeks regarding any stressful event from their past. Likert-type responses go from "Never" to "9 times or more". To consider whether a participant had clinically-significant symptoms of PTSD, she/he had to meet the above criteria and score above 24 points.

The Spence Children's Anxiety Scale-Spanish version (SCAS) [27] [28] was used to assess the severity of anxiety symptoms in relation to anxiety disorder as measured by the DSM-IV. This scale assesses a total score based on 6 sub-domains of anxiety, namely: generalized anxiety, panic/agoraphobia, social phobia, separation anxiety, obsessive-compulsive disorder, and fear of physical injury. The scale presents 44 statements such as, "I fear darkness" or "I'm afraid if I'm away from my parents", to which subjects are asked to respond on a scale that runs from "Never" to "Always". Significant clinical symptomatology is considered for scores of 65 and above.

\subsection{Analysis}

As mentioned above, the final analysis included 10 institutionalized and 10 never-institutionalized adolescents. A t-test for psychopathological scores between groups was performed. In the EEG analyses, a t-test for the RP values of each electrode recorded in each frequency band was carried out. The same analysis was used in the correlation tests for each pair of interhemispheric (Fp1-Fp2; F3-F4) and intrahemispheric derivations (Fp1-F3, Fp2-F4, F3-T3, F4-T4, F3-P3, F4-P4). Also, in order to determine the relation between EEG characteristics and psychopathological symptoms in each group, Pearson correlation analyses between EEG parameters and participants' CPSS, SCAS and CDI scores were performed for the entire sample.

\section{Results}

\subsection{Psychopathological Scores}

Participants' scores on the CDI for the sub-domain dysphoria and the total score and sub-domain scores for re-experimentation and arousal on the CPSS were significantly higher in IA than NIA, though no significant between-group differences appeared on the SCAS. Table 2 shows the means, standard deviations and number of participants in each group that obtained clinically-significant scores on the different scales and sub-domains. That table also presents the $t$ and $p$ values, as well as the size effect of the statistical comparisons.

\subsection{Electroencephalographic Data}

The IA group showed higher RP in the theta $(t=2.05, p=0.05$, Cohen's $d=$ $0.435)$ and alpha1 $(t=2.98, p=0.008$, Cohen's $d=0.568)$ bands at the right frontopolar (Fp2) derivations than NIA. Also, IA presented lower RP in the faster bands: bilaterally in betal in the frontopolar $(\mathrm{Fp} 1 \mathrm{t}=-2.095, \mathrm{p}=0.05$, Cohen's $\mathrm{d}=0.442$; Fp2 $\mathrm{t}=-2.099, \mathrm{p}=0.05$, Cohen's $\mathrm{d}=0.443$ ) and dorsolateral 
Table 2. Behavioural scores. Mean and standard error for the institutionalized (IA) and never-institutionalized (NIA) groups of adolescents obtained from the CDI: Child Depression Index; CPSS: Children's Post-Traumatic Stress Scale; and SCAS: Spence Children's Anxiety Scale. The number of participants that presented clinically-significant scores is specified. Clinically Significant Scores IA/NIA (CSS IA/NIA).

\begin{tabular}{|c|c|c|c|c|c|c|c|c|}
\hline \multirow{2}{*}{ Psychopathological scales } & \multicolumn{2}{|c|}{ IA $(n=10)$} & \multicolumn{2}{|c|}{ NIA $(n=10)$} & \multicolumn{2}{|c|}{ Comparisons } & \multirow[t]{2}{*}{ Cohen's d } & \multirow{2}{*}{ - CSS IA/NIA } \\
\hline & M & S.E. & M & S.E. & $\mathrm{t}$ & $p$ & & \\
\hline \multicolumn{9}{|l|}{ CDI } \\
\hline Total & 48.3 & \pm 2.34 & 42.11 & \pm 2.24 & 1.826 & 0.085 & 0.394 & $0 / 0$ \\
\hline Dysphoria & 52.6 & 1.63 & 42.44 & \pm 1.99 & 3.979 & $0.001^{*}$ & 0.683 & $0 / 0$ \\
\hline Low self-esteem & 45.7 & 3.01 & 44.44 & \pm 2.75 & 0.306 & 0.763 & 0.071 & $0 / 0$ \\
\hline \multicolumn{9}{|l|}{ CPSS } \\
\hline Total & 18.3 & \pm 5.24 & 5 & \pm 1.8 & 2.402 & $0.035^{*}$ & 0.492 & $3 / 0$ \\
\hline Re-experimentation & 6.2 & \pm 1.67 & 1.5 & \pm 0.528 & 2.665 & $0.022^{\star}$ & 0.531 & $4 / 0$ \\
\hline Arousal & 4.8 & \pm 1.54 & 4.87 & \pm 0.517 & 2.449 & $0.037^{*}$ & 0.498 & $5 / 0$ \\
\hline Avoidance & 6.8 & \pm 2.3 & 1.8 & \pm 0.8 & 2.054 & 0.064 & 0.435 & $5 / 0$ \\
\hline \multicolumn{9}{|l|}{ SCAS } \\
\hline Total & 59.2 & \pm 2.021 & 51.2 & \pm 4.09 & 1.754 & 0.097 & 0.381 & $3 / 1$ \\
\hline Obsessive compulsive disorder & 62.2 & \pm 2.107 & 56 & \pm 13.52 & 4.28 & 0.210 & 0.71 & $3 / 3$ \\
\hline Social phobia & 57 & \pm 2.35 & 50 & \pm 4.28 & 1.499 & 0.151 & 0.331 & $2 / 2$ \\
\hline Panic/agoraphobia & 55.4 & \pm 3.53 & 55.3 & \pm 4.33 & 0.018 & 0.986 & 0.004 & $2 / 4$ \\
\hline Separation anxiety & 58.2 & \pm 3.35 & 59.2 & \pm 2.55 & -0.24 & 0.815 & 0.056 & $4 / 4$ \\
\hline Physical injury & 50.2 & \pm 3.1 & 53 & \pm 2.81 & -0.67 & 0.512 & 0.155 & $2 / 1$ \\
\hline Generalized anxiety & 57 & \pm 2.18 & 54.5 & \pm 3.13 & 0.656 & 0.520 & 0.152 & $1 / 1$ \\
\hline
\end{tabular}

frontal areas (F3 t $=-2.19, \mathrm{p}=0.041$, Cohen's $\mathrm{d}=0.46 ; \mathrm{F} 4 \mathrm{t}=-2.08, \mathrm{p}=0.042$, Cohen's $\mathrm{d}=0.458$ ), as well as in alpha2 in the left dorsolateral frontal (F3) area ( $\mathrm{t}$ $=-2.51, \mathrm{p}=0.021$, Cohen's $\mathrm{d}=0.5)$. IA also had lower RP in the alpha2 band at both temporal lobes (T3 t $=-3.001, \mathrm{p}=0.007$, Cohen's $\mathrm{d}=0.577 ; \mathrm{T} 4 \mathrm{t}=-2.17, \mathrm{p}$ $=0.043$, Cohen's $\mathrm{d}=0.455)$ (Figure 1).

IA participants presented a higher interhemispheric correlation in the alpha1 and alpha2 bands between frontopolar regions (Fp1-Fp2) than NIA (Figure 2). The $t$ and $p$ values of the comparisons are presented in Table 3.

IA also had a significantly-lower correlation in the left hemisphere between the dorsolateral and parietal (F3-P3), and dorsolateral and temporal areas (F3-T3), specifically in the gamma band (Figure 2). The $t$ and $p$ values of the comparisons are presented in Table 3.

In terms of intrahemispheric correlation, the IA group showed a higher correlation between the frontopolar and dorsolateral prefrontal areas in the delta, theta and alpha1 bands in the left (Fp1-F3) and right hemispheres (Fp2-F4), 
though in this case only in the alpha 1 band. Similarly, there was a higher EEG correlation between the left frontopolar and parietal derivations (Fp1-P3) in the delta and beta1 bands in the IA group compared to NIA.

\subsection{Correlation between EEG Parameters and Psychopathological Scores}

A negative correlation between the SCAS total scores and RP in T3 $(r=-0.606$,

\section{Relative Power}

Fp1

Fp2

$\square$ IA
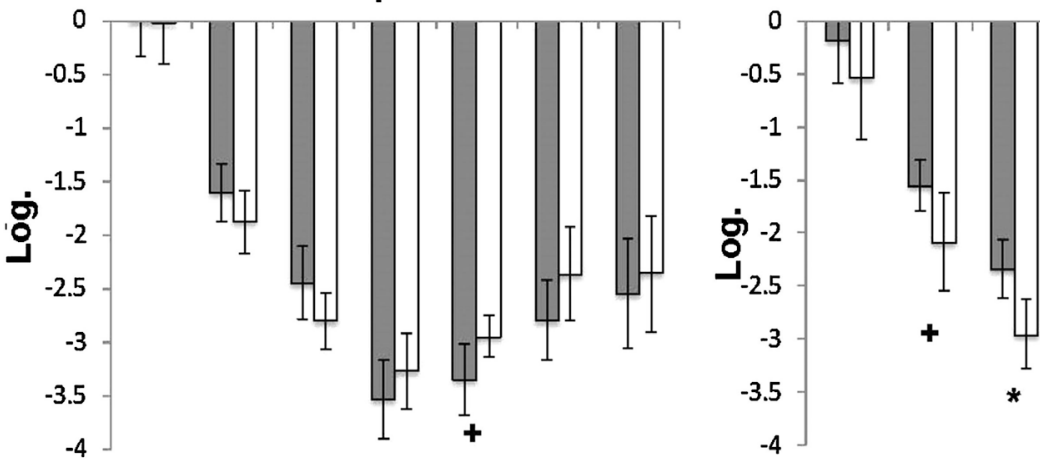

$\square$ NIA

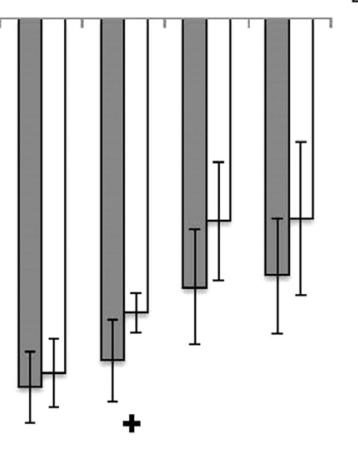

F3

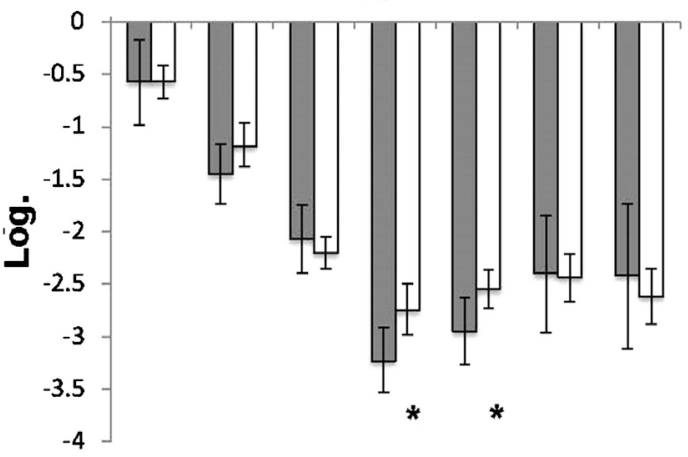

T3

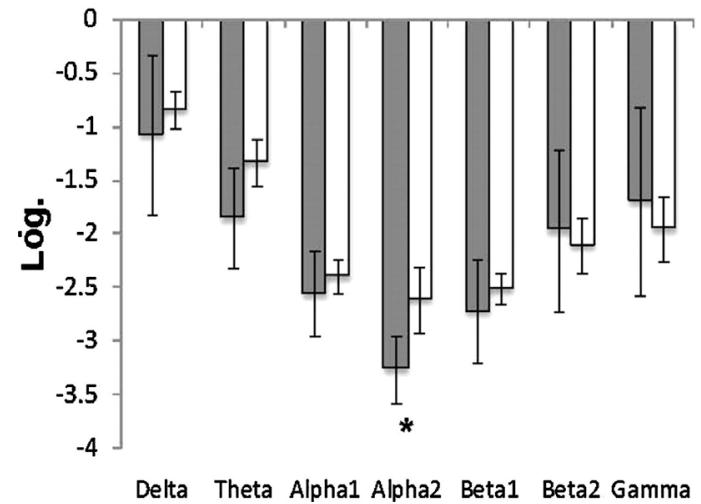

F4

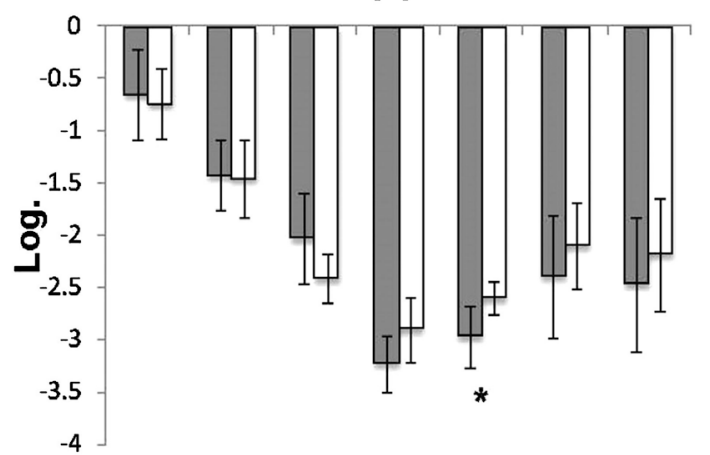

T4

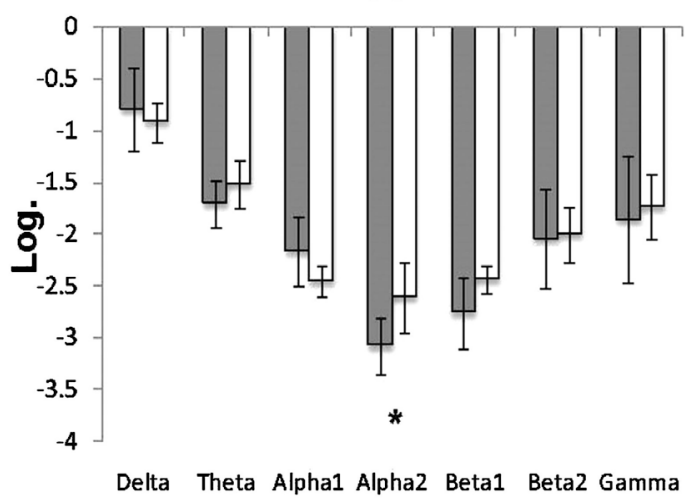

Figure 1. Relative power converted to logarithms (mean \pm 1 standard error) for the institutionalized (IA) and never-institutionalized adolescents (NIA), from the derivations at which there were significant between-group differences. ${ }^{*} \mathrm{p}<0.05 ;^{+}=0.05$. 


\section{EEG Inter and Intrahemispheric Correlations}
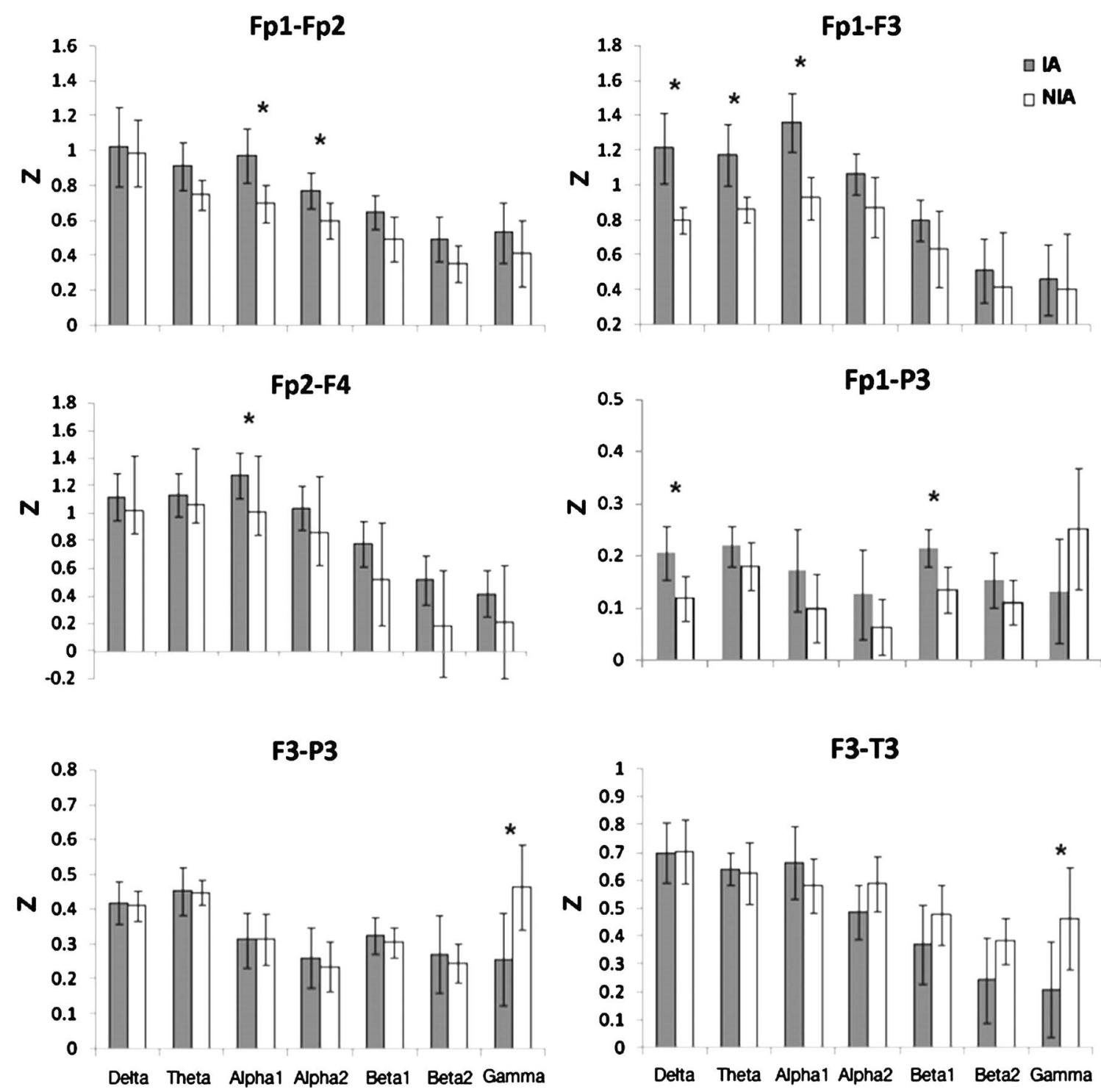

Figure 2. EEG correlations converted to $\mathrm{Z}$ (mean \pm 1 standard error) for the institutionalized (IA) and never-institutionalized adolescents (NIA), from the derivations at which there were significant between-group differences. ${ }^{*} \mathrm{p}<0.05$.

$\mathrm{p}=0.005)$ and $\mathrm{T} 4(\mathrm{r}=-0.462, \mathrm{p}=0.040)$ areas and SCAS OCD scores and alpha2 RP in T3 $(r=-0.658, p=0.002)$ and $T 4(r=-0.547, p=0.013)$ areas was found. The OCD SCAS also presented a negative correlation with the right dorsolateral frontal (F4) RP in alpha2 $(r=-0.500, p=0.025)$. In addition, a negative correlation was found between total CPSS $(r=-0.446, p=0.049)$, re-experiencing CPSS $(r=-0.496, p=0.026)$, and dysphoria CDI scores $(r=-0.494, p=0.032)$ with the right frontopolar prefrontal RP (Fp2) in beta1. Moreover, CDI total scores had a negative correlation with T3 alpha2 band $(r=-0.533, p=0.019)$.

As Table 4 shows, re-experiencing, avoidance and arousal CPSS scores showed a positive correlation with the inter-frontopolar-prefrontal EEG correlation 
Table 3. EEG correlations. Significant EEG correlation differences between an institutionalized (IA) and a never-institutionalized (NIA) group of adolescents. IA > NIA means that the EEG correlations were higher in IA than in NIA. IA < NIA means the opposite.

\begin{tabular}{|c|c|c|c|c|c|c|c|c|}
\hline EEGr & IA $>$ NIA & $\mathrm{t}$ & $\mathrm{p}$ & Cohen's d & IA $<$ NIA & $\mathrm{t}$ & $\mathrm{p}$ & Cohen's d \\
\hline \multirow{2}{*}{ Fp1-Fp2 } & Alpha-1 & 2.85 & 0.01 & 0.558 & \multirow{2}{*}{---} & & & \\
\hline & Alpha-2 & 2.29 & 0.03 & 0.475 & & & & \\
\hline F3-F4 & --- & & & & --- & & & \\
\hline \multirow{3}{*}{ Fp1-F3 } & Delta & 3.91 & 0.001 & 0.677 & \multirow{3}{*}{---} & & & \\
\hline & Theta & 3.26 & 0.004 & 0.61 & & & & \\
\hline & Alpha-1 & 4.17 & 0.0005 & 0.7 & & & & \\
\hline Fp2-F4 & Alpha-1 & 2.24 & 0.037 & 0.468 & --- & & & \\
\hline \multirow{2}{*}{ FP1-P3 } & Delta & 2.57 & 0.019 & 0.519 & \multirow{2}{*}{---} & & & \\
\hline & Betal & 2.86 & 0.01 & 0.559 & & & & \\
\hline Fp2-P4 & --- & & & & --- & & & \\
\hline F3-P3 & --- & & & & Gamma & -2.3 & 0.033 & 0.478 \\
\hline F3-P4 & --- & & & & --- & & & \\
\hline F3-T3 & --- & & & & Gamma & -2.01 & 0.05 & 0.429 \\
\hline $\mathrm{F} 4-\mathrm{T} 4$ & --- & & & & --- & & & \\
\hline
\end{tabular}

Table 4. EEG-behavioural Pearson correlations. Significant Pearson correlations between EEG correlations and the CDI, CPSS and SCAS scores.

\begin{tabular}{|c|c|c|c|c|c|c|c|c|c|c|c|c|c|c|}
\hline \multirow[t]{2}{*}{ Behaviour/EEG $r$} & \multicolumn{2}{|c|}{$\begin{array}{c}\text { Fp1-Fp2 } \\
\text { Alpha1 }\end{array}$} & \multicolumn{2}{|c|}{$\begin{array}{c}\text { Fp1-Fp2 } \\
\text { Alpha2 }\end{array}$} & \multicolumn{2}{|c|}{$\begin{array}{l}\text { Fp1-F3 } \\
\text { Delta }\end{array}$} & \multicolumn{2}{|c|}{$\begin{array}{l}\text { Fp1-F3 } \\
\text { Theta }\end{array}$} & \multicolumn{2}{|c|}{$\begin{array}{l}\text { Fp1-F3 } \\
\text { Alpha1 }\end{array}$} & \multicolumn{2}{|c|}{$\begin{array}{l}\text { Fp2-F4 } \\
\text { Alpha1 }\end{array}$} & \multicolumn{2}{|c|}{$\begin{array}{c}\text { F3-T3 } \\
\text { Gamma }\end{array}$} \\
\hline & $\mathrm{r}$ & $\mathrm{p}$ & $\mathrm{r}$ & $\mathrm{p}$ & $\mathrm{r}$ & $\mathrm{p}$ & $\mathrm{r}$ & $\mathrm{p}$ & $\mathrm{r}$ & $\mathrm{p}$ & $\mathrm{r}$ & $\mathrm{p}$ & $\mathrm{r}$ & $\mathrm{p}$ \\
\hline CPSS Re-experiencing & 0.625 & 0.003 & & & & & & & 0.522 & 0.018 & & & & \\
\hline Avoidance & 0.599 & 0.005 & 0.447 & 0.048 & & & & & & & 0.455 & 0.044 & & \\
\hline Arousal & 0.540 & 0.014 & & & & & & & & & & & & \\
\hline \multicolumn{15}{|l|}{ SCAS } \\
\hline Panic agoraphobic & & & & & & & & & & & & & 0.482 & 0.032 \\
\hline Separation anxiety & & & & & & & & & & & & & 0.452 & 0.045 \\
\hline CDI Dysphoria & & & & & 0.520 & 0.023 & 0.494 & 00.032 & 00.577 & 0.010 & 0.540 & 0.017 & & \\
\hline
\end{tabular}

(Fp1-Fp2). Avoidance CPSS presented a positive correlation with the alpha2 inter-frontopolar (Fp1-Fp2) and left intra-frontal (Fp1-F3) EEG correlations, while re-experiencing CPSS showed a positive correlation with the left intra-frontal EEG correlation (Fp1-F3) in alpha1. Panic agoraphobic and separation anxiety SCAS scores presented positive correlations with the left fronto-temporal EEG correlation in gamma. Finally, dysphoria CDI scores showed a positive correlation with the EEG correlation in the pair of derivations Fp1-F3 in the delta, theta and alpha1 bands, as well as at Fp2-F4 in alpha1 (Table 4). 


\section{Discussion}

The results of this study indicate that adolescents who had been institutionalized for over $75 \%$ of their lifetime presented an electrical organization of the brain distinct from that of the subjects who had lived their entire lives in family settings with their parents. Some of the EEG parameters assessed showed correlations with anxiety, PTSD and dysphoric symptoms. In addition, the institutionalized adolescents showed greater symptoms of PTSD and dysphoria, and more of them presented clinically-significant symptoms of anxiety and PTSD.

\subsection{Psychopathological Scores}

The institutionalized adolescents had higher scores for dysphoria (CDI) and PTSD than those in the NIA group. Also, more of them presented clinically-significant symptoms of anxiety and PTSD. Several authors have established a relation between the time of institutionalization and the onset of mental disorders [7] [29]. Thus, the higher scores for depression and PTSD in the IA group could be the result of the lack of trustworthy, long-term social bonds and the scarcity of alternatives for dealing with stressful situations; factors that could induce the impairments in the socio-emotional interactions of such subjects that have been reported as characteristic of institutional care [30]. These features might emanate from the lack of social and personal interaction between a child and her/his caretaker called "serve and return", which sustains normal neurodevelopment in children [31]. This finding concurs with studies, which mention that neglect is even more strongly-related to depression outcomes than sexual or physical abuse [32]. Although physical and emotional neglect partially contribute to PTSD symptoms, there are other types of maltreatment that contribute more to PTSD, such as emotional or sexual abuse [33]. Therefore, we cannot rule out that the higher intensity of PTSD symptoms could be related to stressful situations experienced by adolescents during, or before, their institutionalization.

\subsection{EEG Activity and Psychopathological Scores}

EEG activity in different areas in adolescents might reflect abnormal development or lack of development. Regarding EEG Relative Power for example, the negative correlation between Fp2 RP in the beta 1 band and CPSS (PTSD) and CDI dysphoria could be related to cortical excitability, since similar beta frequencies have been found in insomnia patients [34]. Hence, restlessness might be a facilitator of this increase, given that high frequencies such as beta (or gamma) waves reflect cortical activation [35] and so might also reflect behavioural arousal [36] [37] [38]. We consider that this finding was driven by the higher beta1 band found in the IA group at the Fp1, Fp2 and F4 electrodes, since this finding proved to be significant when the IA group was analyzed independently. On the other hand, regarding the F2 electrode, it has been reported that EEG frequencies become faster with age [39]. While the RP of slow bands like 
theta and alphal decreases, the RP of beta increases with age in early adolescents with normal development and no signs of depression and anxiety [40] [41]. Also, there are reports of a higher presence of theta power in learning-disabled children compared to normal children, and in institutionalized children compared to never-institutionalized populations at the aforementioned derivations. These findings have been related to a pattern of immaturity [11] [42]. Therefore, it is possible that the increased theta RP observed in IA could be associated with a pattern of immaturity, as has been reported by other authors.

These results, which are common to a variety of disorders, including ADHD and autism, have also been described as signaling deviations in brain development [11]. This was best expressed when Corning et al. [43], demonstrated a negative correlation between the theta and alpha bands and Wechsler subscales like arithmetic, picture completion, picture arrangement, and object assembly, and went on to explain that these bands indicate a pattern of maturation. Although we did not find any parametric correlation among our Fp1 and F4 beta1 EEG correlation and psychopathological scores, we assume that the explanation for these findings is related to this explanation of cortical excitability.

Other findings that might be related to abnormal development are from the $\mathrm{RP}$ of the F3 area in relation to the alpha2 and betal bands. In this regard, a lower proportion of alpha 2 and beta1 bands in temporal and frontal areas has been documented in resistant schizophrenic patients compared to non-resistant patients and controls [44] as in our results. Those authors explain their findings on EEG RP by suggesting a relation to various morphological and physiological abnormalities. This would be in line with the explanation of our EEG relative power findings based on the lack of different types of stimuli that could provoke, as well, abnormal development of cortical areas with subsequent differences in electrical features [10]. This relationship could be confirmed by our negative relationship between $\mathrm{F} 3$ in the alpha2 band and psychopathological scores.

Additionally, we observed a negative association between T3 and T4 in the alpha2 band and anxiety scores (SCAS Total score and SCAS CDI). This correlation could be related to the limbic irritability described by Teicher [45] in children exposed to early stress. The emergence of temporal lobe or limbic seizure-like activity-"limbic irritability" - could be a consequence (as has been found in studies of early stress in animals) of the reduced density of both central benzodiazepine receptors and high-affinity GABA-A receptors [46]. Again, we consider that this finding was driven by the IA group since it was there that we found the same $\mathrm{T} 3$ and $\mathrm{T} 4$ alpha2 results.

Considering that we obtained a negative correlation between the alpha2 band and anxiety scores in temporal areas, we conclude that the low RP of alpha2 reflects higher activity in temporal areas and so generates higher anxiety scores. Conversely, the higher RP of alpha2 reveals lower activity in temporal areas, which led to lower anxiety scores.

Regarding our EEG results, we found a positive relation between frontopolar 
EEG correlations and anxiety scores. First, there is a significant decrease in frontopolar thickness between 8- and 20-year-olds [47]. Discussions of functionality within frontopolar connectivity have reported that functional interhemispheric coupling decreases with age, though it does not occur in other areas, such as the parietal, temporal or occipital regions [48]. Functionally, a higher interhemispheric hemodynamic coherence between frontopolar cortices in autistic children with low social abilities during a rest condition has been found [49]. If we consider reports that have established the importance of frontal-polar areas in the state-dependent regulation of emotion [50], then perhaps basal functional coupling is a mechanism that helps institutionalized adolescents cope with stressful situations. Considering that institutional populations have been described as having a quasi-autistic syndrome [9], perhaps this higher functional coupling between frontopolar cortices is a common characteristic of autistic and institutionalized populations, and is related to abnormal connection pruning.

We also found a positive correlation between functional connectivity among prefrontal regions (Fp1, Fp2, F3 and F4) in the alpha bands and CPSS subscales like re-experiencing, avoidance and arousal. Similar results emerged from the CDI dysphoria subscale, where a positive correlation between functional connectivity among prefrontal regions in the delta, theta and alpha bands was identified. It has been suggested that brain structures like the prefrontal cortex are particularly vulnerable to stress, since they have a high density of glucocorticoid receptors and experience significant postnatal development [45]. On the other hand, there is evidence to suggest that decreased coupling among brain areas is related to optimal organization of cortical networks, such that increases may indicate immaturity and a lack of cortical specialization [51]. This argument might be based on explanations which propose that short-distance connectivity is critical in generating functional specificity (information) [52].Upon analysing our dysphoria-EEG correlation we realized that the main findings occurred in the left prefrontal hemisphere with one positive correlation in the right hemisphere. In this regard, it has been argued that the Behavioural Activation System (BAS system) is related primarily to left hemisphere activation during approach motivational situations, but to the right hemisphere under withdrawal motivation [53]. We consider that the increased basal correlation among left frontal areas in the slow bands (delta, theta and alpha1) could indicate a less effective mechanism and, therefore, the propensity to present dysphoria. Under these assumptions, this left activity could be related to a lack of motivation to handle anxious situations under a more psychological context. On the other hand, we must analyze these results, as well, in light of the theory proposed by Higgins (1997), which holds that there are two systems for attaining desired outcomes: one called promotion, the other prevention. Promotion occurs when we "make things happen" in order to obtain a relevant outcome; while prevention acts to "avoid something bad from happening" in order to obtain the desired outcome. 
Perhaps our right interior prefrontal EEG correlation finding is related to a less effective production of motivation to take measures "to prevent things from happening".

Regarding the positive correlation between SCAS and the F3-T3 gamma band EEG-correlation, it is important to recall that those areas are critical for the control and regulation of social behavior [54]. In relation to our results, perhaps the salience of the absence (in social separation) or presence (in panic agoraphobic) of social influence manifested through the fronto-temporal correlation leads to the anxiety scores we recorded. If we consider that long-distance connections allow cortical areas to interact among themselves and so integrate different sources of information into coherent behavioral and cognitive states [17], it would be logical to think that fronto-temporal functional coupling does not represent an efficient way of processing social information.

Turning to the functional connectivity between the prefrontal and posterior cortices, our study found a lower gamma correlation in the left hemisphere between the prefronto-parietal and prefronto-temporal areas in IA. Similar findings have been observed with highly-functional autistic participants using fMRI techniques, which showed a lower correlation between prefrontal and parietal areas compared to controls [55]. Similarly, Brzezicka proposed that the fronto-parietal network plays a fundamental role in integrating information into the coherent mental representations required to resolve problems, and that the lack of such integration could be associated with the presentation of negative moods and depressive states [56]. Considering all these results, we could argue that our findings might be related to an inefficient transit of information between anterior and posterior areas.

Finally, we also found a higher EEG correlation between the anterior prefrontal and parietal areas in the left hemisphere in the delta and beta bands in IA. This EEG correlation again shares some similarities with data from autistic patients reported by Duffy and Als [57], who observed higher coherence between frontopolar and posterior parietal areas compared to controls. Those authors consider that their findings reflect a mechanism in the brain that seeks to compensate for abnormal brain network development. In our IA group, there was no evidence of such a factor that drives abnormal brain development as in autistic groups; however, it could be that an adverse environment drives the development of uncommon brain connectivity.

Finally, it is important to mention that the sample for this project was small due to several factors: the high number of institutionalized adolescents with low IQ, the effects of long-term psychiatric treatment, the few adolescents that had spent $75 \%$ of their lives in orphanages, the few institutions for adolescents (since most house younger children), and the need to remove EEG recordings affected by artefacts. These conditions made it necessary to recruit adolescents from 4 different institutions in 3 states in Mexico. Thus, the features of the study sample itself limited our ability to recruit more participants. 


\section{Conclusion}

In conclusion, the IA adolescents presented more psychopathological symptoms and a brain electrical organization distinct from the controls, which had lived their entire lives with their parents. Their EEG parameters are similar to those of young people with psychiatric disorders like autism, and showed correlations with anxiety, depression and PTSD scores. We believe that these EEG findings can contribute to improving our understanding of the impact that early stress may have on the development of the cortex, and how this may predispose adolescents to develop psychopathologies.

\section{Acknowledgements}

We thank to CONAYCT with the grant 290840 and CB/2012/180981. We also thank to Procuraduría de la Defensa del Menor y la Familia (PRODEMEFA) of the Sistema Integral de la Familia (DIF) of the state of Yucatán, México and to orphan institutions: Comunidad de Restauración Integral de Adolescentes (CRIAI.A.P.), Centro de Atención Integral al Menor en Desamparo (CAIMEDE) in Mérida, Yucatán, Hogar Cabañas, in Guadalajara, Jalisco and Cancún Pro Kids in Cancún Quintana Roo, for their support.

\section{Conflicts of Interest}

The authors declare no conflicts of interest regarding the publication of this paper.

\section{References}

[1] Berens, A.E. and Nelson, C.A. (2015) The Science of Early Adversity: Is There a Role for Large Institutions in the Care of Vulnerable Children? The Lancet, 386, 388-398. https://doi.org/10.1016/S0140-6736(14)61131-4

[2] Nelson, C.A. (2007) A Neurobiological Perspective on Early Human Deprivation. Child Development Perspectives, 1, 13-18. https://doi.org/10.1097/PSY.0000000000000421

[3] Hermenau, K., Goessmann, K., Rygaard, N.P., Landolt, M.A. and Hecker, T. (2016) Fostering Child Development by Improving Care Quality: A Systematic Review of the Effectiveness of Structural Interventions and Caregiver Trainings in Institutional Care. Trauma, Violence, \& Abuse, 18, 544-561. https://doi.org/10.1177/1524838016641918

[4] Bos, K.J. (2009) Effects of Early Psychosocial Deprivation on the Development of Memory and Executive Function. Frontiers in Behavioral Neuroscience, 3, 1-7. https://doi.org/10.3389/neuro.08.016.2009

[5] Smyke, A.T., Koga, S.F., Johnson, D.E., Johnson, D.E., Fox, N.A., Marshall, J., Nelson, C.A., Zeanah, C.H. and the BEIP Core Group (2007) The Caregiving Context in Institution-Reared and Family-Reared Infants and Toddlers in Romania. Journal of Child Psychology and Psychiatry, 48, 210-218.

https://doi.org/10.1111/j.1469-7610.2006.01694.x

[6] McLaughlin, K.A., Sheridan, M.A., Winter, W., Fox, N.A., Zeanah, C.H. and Nelson, C.A. (2014) Widespread Reductions in Cortical Thickness Following Severe Early-Life Deprivation: A Neurodevelopmental Pathway to Attention-Deficit or 
Hyperactivity Disorder. Biological Psychiatry, 76, 629-638.

https://doi.org/10.1016/j.biopsych.2013.08.016

[7] McLaughlin, K.A., Fox, N.A., Zeanah, C.H., Sheridan, M.A., Marshall, P. and Nelson, C.A. (2010) Delayed Maturation in Brain Electrical Activity Partially Explains the Association between Early Environmental Deprivation and Symptoms of Attention-Deficit/Hyperactivity Disorder. Biological Psychiatry, 68, 329-336.

https://doi.org/10.1016/j.biopsych.2010.04.005

[8] Slopen, N., McLaughlin, K.A., Fox, N.A., Zeanah, C.H. and Nelson, C.A. (2012) Alterations in Neural Processing and Psychopathology in Children Raised in Institutions. Archives of General Psychiatry, 69, 1-20.

https://doi.org/10.1001/archgenpsychiatry.2012.444

[9] Nelson III, C.A., Bos, K., Gunnar, M.R. and Sonuga-Barke, E.J.S. (2011) The Neurobiological Toll of Early Human Deprivation. Monographs of the Society for Research in Child Development, 76, 127-146. https://doi.org/10.1111/j.1540-5834.2011.00630.x

[10] Hodel, A.S., Hunt, R.H., Cowell, R.A., Van Den Heuvel, S.E., Gunnar, M.R. and Thomas, K.M. (2015) Duration of Early Adversity and Structural Brain Development in Post-Institutionalized Adolescents. NeuroImage, 105, 112-119. https://doi.org/10.1016/j.neuroimage.2014.10.020

[11] Marshall, P.J., Fox, N.A. and Bucharest Early Intervention Project Core Group (2004) A Comparison of the Electroencephalogram between Institutionalized and Community Children in Romania. Journal of Cognitive Neuroscience, 16, 1327-1338. https://doi.org/10.1162/0898929042304723

[12] Eluvathingal, T.J., Chugani, H.T., Behen, M.E., Juhász, C., Muzik, O., Maqbool, M., Chugani, D.C. and Makki, M. (2006) Abnormal Brain Connectivity in Children after Early Severe Socioemotional Deprivation: A Diffusion Tensor Imaging Study. Pediatrics, 117, 2093-2100. https://doi.org/10.1542/peds.2005-1727

[13] Govindan, R.M., Behen, M.E., Helder, E., Makki, M.I. and Chugani, H.T. (2010) Altered Water Diffusivity in Cortical Association Tracts in Children with Early Deprivation Identified with Tract-Based Spatial Statistics (TBSS). Cerebral Cortex, 20, 561-569. https://doi.org/10.1093/cercor/bhp122

[14] Fries, P. (2005) A Mechanism for Cognitive Dynamics: Neuronal Communication through Neuronal Coherence. Trends in Cognitive Sciences, 9, 474-480. https://doi.org/10.1016/j.tics.2005.08.011

[15] Gevins, A., Leong, H., Du, R., Smith, M.E., Le, J., DuRousseau, D., Zhang, J. and Libove, J. (1995) Towards Measurement of Brain Function in Operational Environments. Biological Psychology, 40, 169-186. https://doi.org/10.1016/0301-0511(95)05105-8

[16] Barttfeld, P., Wicker, B., Cukier, S., Navarta, S., Lew, S. and Sigman, M. (2011) A Big-World Network in ASD: Dynamical Connectivity Analysis Reflects a Deficit in Long-Range Connections and an Excess of Short-Range Connections. Neuropsychologia, 49, 254-263. https://doi.org/10.1016/j.neuropsychologia.2010.11.024

[17] Friston, K.J. (1998) The Disconnection Hypothesis. Schizophrphrenia Research, 30, 115-125. https://doi.org/10.1016/S0920-9964(97)00140-0

[18] Chan, A.S., Sze, S.L. and Cheung, M.C. (2007) Quantitative Electroencephalographic Profiles for Children with Autistic Spectrum Disorder. Neuropsychology, 21, 74-81. https://doi.org/10.1037/0894-4105.21.1.74

[19] Murias, M., Webb, S.J., Greenson, J. and Dawson, G. (2007) Resting State Cortical Connectivity Reflected in EEG Coherence in Individuals with Autism. Biological 
Psychiatry, 62, 270-273. https://doi.org/10.1016/j.biopsych.2006.11.012

[20] Wechsler, D. (2003) WAIS-III: Escala Wechsler de Inteligencia para adultos-III. El Manual Moderno, México.

[21] Wechsler, D. (2003) Escala Wechsler de Inteligencia para Niños-IV. Manual Moderno, México.

[22] Ostrosky-Solis, F., Ardila, A, and Rosselli, M. (1997) NEUROPSI: Evaluación Neuropsicológica Breve en Español. El Manual Moderno, México.

[23] Guevara, M.A., Sanz-Martín, A., Corsi-Cabrera, M., Amezcua-Gutiérrez, C. and Hernández-González, M. (2010) CHECASEN: Programa para revisar señales EEG fuera de línea. Revista mexicana de ingeniería biomédica, 31, 135-141.

[24] Guevara, M.A., Sanz-Martin, A. and Hernández-González, M. (2014) EEGbands: A Computer Program to Statistically Analyze Parameters of Electroencephalographic Signals. Journal of Behavioral Brain Science, 4, 308-324. https://doi.org/10.4236/jbbs.2014.47032

[25] Kovacs, M. (2004) CDI: Inventario de Depresión Infantil. TEA ediciones S.A. de C.V., Madrid.

[26] Bustos, P., Rincón, P. and Aedo, J. (2009) Validación Preliminar de la Escala Infantil de Síntomas del Trastorno de Estrés Postraumático (Child PTSD Symptom Scale, CPSS) en Niños/as y Adolescentes Víctimas de Violencia Sexual. Psykhe, 18, 113-126. https://doi.org/10.4067/S0718-22282009000200008

[27] Spence, S.H., Barrett, P.M. and Turner, C.M. (2003) Psychometric Properties of the Spence Children's Anxiety Scale with Young Adolescents. Journal of Anxiety Disorders, 17, 605-625. https://doi.org/10.1016/S0887-6185(02)00236-0

[28] Orgilés, M., Méndez, X., Spence, S.H., Huedo-Medina, T.B. and Espada, J.P. (2012) Spanish Validation of the Spence Children's Anxiety Scale. Child Psychiatry\& Human Development, 43, 271-281. https://doi.org/10.1007/s10578-011-0265-y

[29] Bos, K., Zeanah, C.H., Fox, N.A., Drury, S.S., McLaughlin, K.A. and Nelson, C.A. (2011) Psychiatric Outcomes in Young Children with a History of Institutionalization. Harvard Review of Psychiatry, 19, 15-24. https://doi.org/10.3109/10673229.2011.549773

[30] Erol, N., Simsek, Z. and Münir, K. (2009) Mental Health of Adolescents Reared in Institutional Care in Turkey: Challenges and Hope in the Twenty-First Century. European Child Adolescent Psychiatry, 19, 113-124. https://doi.org/10.1007/s00787-009-0047-2

[31] National Scientific Council on the Developing Child (2012) The Science of Neglect: The Persistence Absence of Responsive Care Disrupt the Developing Brain. Working Paper, 1-20.

[32] Infura, M.R., Reich, C., Parser, P., Schimmenti, A., Bifulco, A. and Kaess, M. (2017) Associations between Depression and Specific Childhood Experiences of Abuse and Neglect: A Meta-Analysis. Journal of Affective Disorders, 190, 47-55. https://doi.org/10.1016/j.jad.2015.09.006

[33] Sullivan, T.P., Fehon, D.C., Andres-Hyman, R.C., Lipschitz, D.S. and Grilo, C.M. (2006) Differential Relationships of Childhood Abuse and Neglect Subtypes to PTSD Symptom Clusters among Adolescent Inpatients. Journal of Traumatic Stress, 19, 229-239. https://doi.org/10.1002/jts.20092

[34] Perlis, M.L., Merica, H., Smith, M.T. and Giles, D.E. (2001) Beta EEG Activity and Insomnia. Sleep Medicine Reviews, 5, 365-376.

https://doi.org/10.1053/smrv.2001.0151 
[35] Nofzinger, E.A., Price, J.C., Meltzer, C.C., Price, J., Steppe, D.A., Miewld, J.M., Kupfer, D.J. and Moore, R.Y. (2000) Towards a Neurobiology of Dysfunctional Arousal in Depression: The Relationship between Beta EEG Power and Regional Cerebral Glucose Metabolism during NREM Sleep. Psychiatry Research, 98, 71-91. https://doi.org/10.1016/S0925-4927(00)00045-7

[36] Merica, H., Blois, R. and Gaillard, J.M. (1998) Spectral Characteristics of Sleep EEG in Chronic Insomnia. European Journal of Neuroscience, 10, 1826-1834. https://doi.org/10.1046/j.1460-9568.1998.00189.x

[37] Lamarche, C.H. and Ogilvie, R.D. (1997) Electrophysiological Changes during the Sleep Onset Period of Psychophysiological Insomniacs, Psychiatric Insomniacs, and Normal Sleepers. Sleep, 20, 724-733. https://doi.org/10.1093/sleep/20.9.726

[38] Clancy, K., Ding, M., Bernat, E., Schmidt, N.B. and Li, W. (2017) Restless "Rest": Intrinsic Sensory Hyperactivity and Disinhibition in Post-Traumatic Stress Disorder. Brain, 140, 2041-2050. https://doi.org/10.1093/brain/awx116

[39] Michels, L., Muthuraman, M., Lüchinger, R., Martin, E., Anwar, A.R., Raethjen, J., Brandeis, D. and Siniatchkin, M. (2013) Developmental Changes of Functional and Directed Resting-State Connectivities Associated with Neuronal Oscillations in EEG. NeuroImage, 81, 231-242. https://doi.org/10.1016/j.neuroimage.2013.04.030

[40] Clarke, A.R., Barry, R.J., Dupuy, F.E., Heckel, L.D., McCarthy, R., Selikowitz, M. and Johnstone, S.J. (2011) Behavioural Differences between EEG-Defined Subgroups of Children with Attention-Deficit/Hyperactivity Disorder. Clinical Neurophysiology, 122, 1333-1341. https://doi.org/10.1016/j.clinph.2010.12.038

[41] Harmony, T., Marosi, E., Díaz de León, A.E., Becker, J. and Fernández, T. (1990) Effect of Sex, Psychosocial Disadvantages and Biological Risk Factors on EEG Maturation. Electroencephalography and Clinical Neurophysiology, 75, 482-491. https://doi.org/10.1016/0013-4694(90)90135-7

[42] Gasser, T., Rousson, V. and Schreiter Gasser, U. (2003) EEG Power and Coherence in Children with Educational Problems. Journal of Clinical Neurophysiology, 20, 273-282. https://doi.org/10.1097/00004691-200307000-00007

[43] Corning, W.C., Steffy, R.A., Anderson, E. and Bowers, P. (1986) EEG "Maturational Lag” Profiles: Follow-Up Analyses. Journal of Abnormal Child Psychology, 14, 235-249. https://doi.org/10.1007/BF00915443

[44] Ramos, J., Cerdán, L.F., Guevara, M.A., Amezcua, C. and Sanz, A. (2001) Abnormal EEG Patterns in Treatment-Resistant Schizophrenic Patients. International Journal of Neuroscience, 109, 47-59. https://doi.org/10.3109/00207450108986524

[45] Teicher, M. (2002) Scars That Won't Heal: The Neurobiology of Child Abuse. Scientific American, 286, 68-75. https://doi.org/10.1038/scientificamerican0302-68

[46] Caldji, C., Francis, D., Sharma, S., Plotsky, P.M. and Meaney, M.J. (2000) The Effects of Early Rearing Environment on the Development of $\mathrm{GABA}_{\mathrm{A}}$ and Central Benzodiazepine Receptor Levels and Novelty-Induced Fearfulness in the Rat. Neuropsychopharmacology, 22, 219-229. https://doi.org/10.1016/S0893-133X(99)00110-4

[47] O’Donnell, S., Noseworthy, M.D., Levine, B. and Dennis, M. (2005) Cortical Thickness of the Frontopolar Area in Typically Developing Children and Adolescents. NeuroImage, 24, 948-954. https://doi.org/10.1016/j.neuroimage.2004.10.014

[48] Homae, F., Watanabe, H., Otobe, T., Nakano, T., Go, T. and Taga, G. (2010) Development of Global Cortical Networks in Early Infancy. Journal of Neuroscience, 30, 4877-4882. https://doi.org/10.1523/JNEUROSCI.5618-09.2010 
[49] Kikuchi, M., Yoshimura, Y., Shitamichi, K., Ueno, S., Hirosawa, T., Munesue, T., Ono, Y., Tsubokawa, T., Haruta, Y., Oi, M., Niida, Y., Remijin, G.B., Takahashi, T., Suzuki, M., Higashida, H. and Minabe, Y. (2013) Anterior Prefrontal Hemodynamic Connectivity in Conscious 3- to 7-Year-Old Children with Typical Development and Autism Spectrum Disorder. PLOS ONE, 8, e56087.

https://doi.org/10.1371/journal.pone.0056087

[50] Papousek, I. and Schulter, G. (2002) Covariations of EEG Asymmetries and Emotional States Indicate That Activity at Frontopolar Locations Is Particularly Affected by State Factors. Psychophysiology, 39, 350-360. https://doi.org/10.1017/S0048577201393083

[51] Thatcher, R.W., North, D.M. and Biver, C.J. (2008) Development of Cortical Connections as Measured by EEG Coherence and Phase Delays. Human Brain Mapping, 29, 1400-1415. https://doi.org/10.1002/hbm.20474

[52] Sporns, O. and Zwi, J.D. (2004) The Small World of the Cerebral Cortex. Neuroinformatics, 2, 145-162. https://doi.org/10.1385/NI:2:2:145

[53] Harmon-Jones, E. and Sigelman, J. (2001) State Anger and Prefrontal Brain Activity: Evidence That Insult-Related Relative Left-Prefrontal Activation Is Associated with Experienced Anger and Aggression. Journal of Personality and Social Psychology, 80, 797-803. https://doi.org/10.1037/0022-3514.80.5.797

[54] Franzen, E.A. and Myers, R.E. (1973) Neural Control of Social Behavior: Prefrontal and Anterior Temporal Cortex. Neuropsychologia, 11, 141-157. https://doi.org/10.1016/0028-3932(73)90002-X

[55] Just, M.A., Cherkassky, V.L., Keller, T.A., Kana, R.K. and Minshew, N.J. (2007) Functional and Anatomical Cortical Underconnectivity in Autism: Evidence from an FMRI Study of an Executive Function Task and Corpus Callosum Morphometry. Cerebral Cortex, 17, 951-961. https://doi.org/10.1093/cercor/bhl006

[56] Brzezicka, A., Sedek, G., Marchewka, A., et al. (2011) A Role for the Right Prefrontal and Bilateral Parietal Cortex in Four-Term Transitive Reasoning: An fMRI Study with Abstract Linear Syllogism Tasks. Acta Neurobiologiae Experimentales, 71, 479-495.

[57] Duffy, F.H. and Als, H. (2012) A Stable Pattern of EEG Spectral Coherence Distinguishes Children with Autism from Neuro-Typical Controls-A Large Case Control Study. BMC Medicine, 10, 64. https://doi.org/10.1186/1741-7015-10-64 